\title{
Helicity at Photospheric and Chromospheric Heights
}

\author{
S. K. Tiwari ${ }^{1}$, P. Venkatakrishnan ${ }^{1}$ and K. Sankarasubramanian ${ }^{2}$ \\ 1 Udaipur Solar Observatory (Physical Research Laboratory), Udaipur, India \\ 2 Space Astron. \& Instrument. Div., ISRO Satellite Center, Bangalore, India
}

\begin{abstract}
Summary. In the solar atmosphere the twist parameter $\alpha$ has the same sign as magnetic helicity. It has been observed using photospheric vector magnetograms that negative/positive helicity is dominant in the northern/southern hemisphere of the Sun. Chromospheric features show dextral/sinistral dominance in the northern/southern hemisphere and sigmoids observed in X-rays also have a dominant sense of reverse-S/forward-S in the northern/southern hemisphere. It is of interest whether individual features have one-to-one correspondence in terms of helicity at different atmospheric heights. We use UBF $\mathrm{H} \alpha$ images from the Dunn Solar Telescope (DST) and other $\mathrm{H} \alpha$ data from Udaipur Solar Observatory and Big Bear Solar Observatory. Near-simultaneous vector magnetograms from the DST are used to establish one-to-one correspondence of helicity at photospheric and chromospheric heights. We plan to extend this investigation with more data including coronal intensities.
\end{abstract}

\section{Introduction}

Helicity is a physical quantity that measures the degree of linkage and twistedness in the field (Berger \& Field 1984). It is derived from a volume integral over the scalar product of the magnetic field $\mathbf{B}$ and its vector potential $\mathbf{A}$. Direct calculation of helicity is not possible due to the non-uniqueness of the vector potential $\mathbf{A}$ and the limited availability of data sampling different layers of the solar atmosphere. The force-free parameter $\alpha$ estimates one component of helicity, i.e., twist, the other component being writhe which can not be derived from the available data. This $\alpha$ is a measure of degree of twist per unit axial length. It has the same sign as magnetic helicity (Pevtsov et al. 2008, Pevtsov 2008). It is now well known that negative/positive helicity dominates in the northern/southern hemisphere. For active regions the hemispheric helicity rule holds in the photosphere, see Hagino \& Sakurai (2005) and references therein. Similarly for the chromospheric and coronal helicity rules, see Bernasconi et al. (2005) and references therein, and Pevtsov et al. 
(2001) and references therein. The topology of chromospheric and coronal features decide the sign of the associated helicity. Chirality is the term used for the sign of the helicity in these features. Thus, helicity is a physical measure of chirality. The chirality of active region features shows correspondence with the sign of the helicity in the associated lower/upper atmospheric features. For example, the chirality of X-ray features with $\mathrm{S}$ (inverse-S) shapes are associated with sinistral (dextral) filaments (Martin 2003, Rust 2003). Chae (2000) reported for a few cases that active filaments showing dextral/sinistral chirality are related with negative/positive magnetic helicity. Pevtsov et al. (2001) demonstrated correspondence between photospheric and coronal chirality for a few active regions. However, this needs to be confirmed. We have reported similar helicity signs at photospheric, chromospheric, and coronal heights for a few active regions (Tiwari et al. 2008).

Comparison between magnetic helicity signs at different heights in the solar atmosphere may be a useful tool to predict solar eruptions leading to interplanetary events. Also, it may help to constrain modeling chromospheric and coronal features taking the photosphere as boundary condition. However, the data required to do this are not directly available and are often non-conclusive. Vector magnetic fields are not available as routinely as is necessary to derive photospheric twist values. Chromospheric $\mathrm{H} \alpha$ images may be available most of the time by combining data from different telescopes, but are not always conclusive due to lack of angular resolution. Analysis of coronal loop observations is required to determine coronal helicity signs, but these are also not available routinely. Above all, it is hard to find data taken simultaneously at different heights in the solar atmosphere. In this work we combine photospheric and chromospheric data from multiple solar observatories and telescopes. They were often not taken at precisely the same time. We therefore assume that the sign of the magnetic helicity does not change within a few hours.

\section{Sign of Magnetic Helicity}

The sign of helicity in the photosphere is usually found from the force-free parameter $\alpha$, e.g., $\alpha_{\text {best }}$ (Pevtsov et al. 1995), averaged $\alpha$, e.g., $\left\langle\alpha_{z}\right\rangle=$ $<\mathbf{J}_{\mathbf{z}} / \mathbf{B}_{\mathbf{z}}>$ (Pevtsov et al. 1994) with current density $\mathbf{J}_{\mathbf{z}}=\nabla \times \mathbf{B}_{\mathbf{z}}$, where $\mathbf{B}_{\mathbf{z}}$ is the vertical component of the magnetic field. Some authors have used the current helicity density $H_{c}=\mathbf{B}_{\mathbf{z}} \cdot \mathbf{J}_{\mathbf{z}}$ (Bao \& Zhang 1998; Hagino \& Sakurai 2005). A good correlation was found between $\alpha_{\text {best }}$ and $\left\langle\alpha_{z}\right\rangle$ by Burnette et al. (2004) and Leka et al. (1996). The force-free parameter $\alpha$ has the same sign as magnetic helicity (Pevtsov et al. 2008). Also, the current helicity (which is not a conserved quantity like magnetic helicity) has the same sign as that of magnetic helicity (Seehafer 1990; Hagyard \& Pevtsov 1999; Pevtsov 2008; Sokoloff et al. 2008). In this study, we use the sign of the global $\alpha$ parameter as the sign of magnetic helicity, giving the twist present in the active region. 
Numerical measurement of the sign of the chromospheric magnetic helicity is not possible due to non-availability of vector magnetic field observations at these heights. However, the twist present in morphological intensity features were reported already long ago (Hale 1925; Richardson 1941) to tend to follow the hemispheric helicity rule, independent of the solar cycle. Later, many researchers studied the chirality of different chromospheric features such as filaments, fibrils, filament channels etc. (Martin 1998, 2003). We use the chirality of these chromospheric features, mostly whirls observed in $\mathrm{H} \alpha$, to derive its association with the photospheric sign of magnetic helicity.

\section{Data Sets Used}

Apart from a few data sets, most are obtained from different solar observatories and telescopes due to the unavailability of all required data from a at the same place. The vector magnetic field data were obtained from the Advanced Stokes Polarimeter (ASP: Elmore et al. 1992) as well as the Diffraction Limited Spectro-polarimeter (DLSP: (Sankarasubramanian et al. 2004, 2006)) both at the DST. Near-simultaneous $\mathrm{H} \alpha$ images from the Universal Bi-refringent Filter (UBF) at the DST are used whenever obtained along with the ASP and DLSP. For the vector field observations which do not have corresponding UBF data, $\mathrm{H} \alpha$ images from Udaipur Solar Observatory (USO) and Big Bear Solar Observatory (BBSO) were used. We made sure that in these cases the $\mathrm{H} \alpha$ images were collected within less than a day. Standard and well-established processing was done to derive vector fields. The procedure is described in the references given above.

\section{Results and Discussion}

Table 1 shows how the sign of helicity at the photospheric level and of the chirality in associated features at chromospheric heights are related with each other. Figure 1 (a) and (b) clearly show that the $\mathrm{H} \alpha$ whirls follow the transverse magnetic field vectors measured at photospheric heights. The positive/negative helicity derived from the global twist in this sunspot are in the photospheric vector data is directly associated with the sinistral/dextral chirality derived from the chromospheric $\mathrm{H} \alpha$ data.

In this preliminary analysis, we thus conclude that the sign of helicity (positive/negative) derived from global twist present around sunspots in the photosphere has one-to-one correspondence with the (sinistral/dextral) sense of chirality observed in the associated chromospheric data. We mostly use the chirality of chromospheric whirls to derive the chromospheric helicity sign. It is known (Martin 1998, 2003) that filaments, filament channels, etc., have the same sense of chirality as the whirls above the associated active regions. The chirality of filaments associated with an active region can therefore be used to 

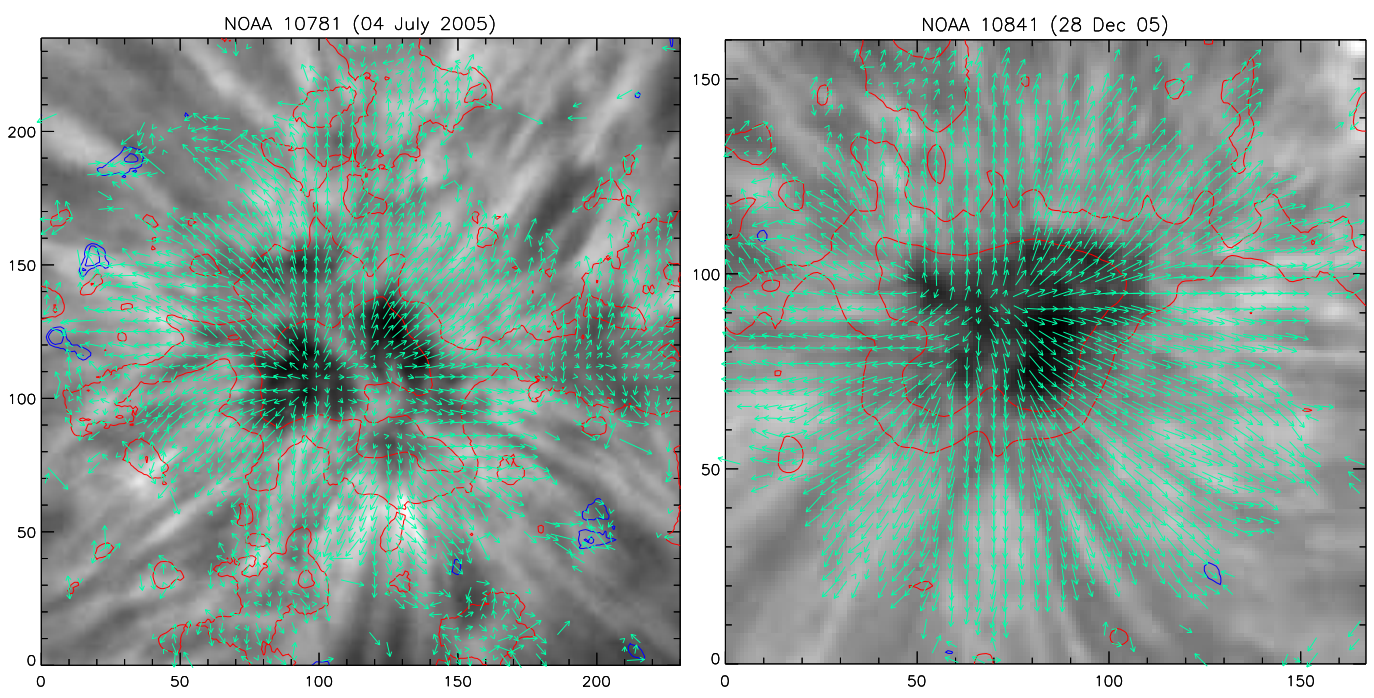

Fig. 1. Two examples of the chromospheric sunspots, with the photospheric transverse vectors of the same field of view over-plotted on them. The axis divisions are pixel numbers. The contours and vectors show longitudinal and transverse magnetic fields, respectively.

Table 1. Sign of helicity at the photospheric and the chromospheric level

\begin{tabular}{cccc}
\hline Observation date & AR NOAA number Photospheric helicity Chromospheric chirality \\
\hline 06 Feb 2007 & NOAA 10941 & Negative & Dextral \\
09 Jan 2007 & NOAA 10935 & Negative & Dextral \\
11 Dec 2006 & NOAA 10930 & Negative & Dextral \\
28 Dec 2005 & NOAA 10841 & Positive & Sinistral \\
22 Dec 2005 & NOAA 10838 & Positive & Sinistral \\
27 Aug 2005 & NOAA 10804 & Positive & Sinistral \\
28 Aug 2005 & NOAA 10803 & Positive & Sinistral \\
26 Aug 2005 & NOAA 10800 & Positive & Sinistral \\
04 July 2005 & NOAA 10781 & Positive & Sinistral \\
17 Apr 2005 & NOAA 10752 & Positive & Sinistral \\
09 Apr 2003 & NOAA 10330 & Positive & Sinistral \\
03 Sep 2001 & NOAA 09601 & Negative \\
01 Sep 2001 & NOAA 09601 & Negative & Dextral \\
30 Aug 2001 & NOAA 09601 & Negative & Dextral \\
01 Sep 2001 & NOAA 09596 & Positive & Sinistral \\
30 Aug 2001 & NOAA 09596 & Positive & Sinistral \\
30 Aug 2001 & NOAA 09591 & Positive & Sinistral \\
26 Aug 2001 & NOAA 09590 & Negative & Dextral \\
24 Aug 2001 & NOAA 09590 & Negative & Dextral \\
24 Aug 2001 & NOAA 09585 & Positive & Sinistral \\
\hline
\end{tabular}

$\sharp$ : dominant sense 
determine the chromospheric sense of chirality when high resolution $\mathrm{H} \alpha$ data are not available.

Acknowledgement. We thank the conference organisers for a very good meeting and the editors for excellent instructions.

\section{References}

Bao, S. Zhang, H. 1998, ApJ, 496, L43+

Berger, M. A. Field, G. B. 1984, Journal of Fluid Mechanics, 147, 133

Bernasconi, P. N., Rust, D. M., Hakim, D. 2005, Solar Phys., 228, 97

Burnette, A. B., Canfield, R. C., Pevtsov, A. A. 2004, ApJ, 606, 565

Chae, J. 2000, ApJ, 540, L115

Elmore, D. F., Lites, B. W., Tomczyk, S., et al. 1992, in Society of Photo-Optical Instrumentation Engineers (SPIE) Conference Series, eds. D. H. Goldstein \& R. A. Chipman, Society of Photo-Optical Instrumentation Engineers (SPIE) Conference Series, 1746, 22

Hagino, M. Sakurai, T. 2005, PASJ, 57, 481

Hagyard, M. J. Pevtsov, A. A. 1999, Solar Phys., 189, 25

Hale, G. E. 1925, PASP, 37, 268

Leka, K. D., Canfield, R. C., McClymont, A. N., van Driel-Gesztelyi, L. 1996, ApJ, 462,547

Martin, S. F. 1998, in IAU Colloq. 167: New Perspectives on Solar Prominences, eds. D. F. Webb, B. Schmieder, \& D. M. Rust, Astronomical Society of the Pacific Conference Series, 150, 419

Martin, S. F. 2003, Advances in Space Research, 32, 1883

Pevtsov, A. A. 2008, Journal of Astrophysics and Astronomy, 29, 49

Pevtsov, A. A., Canfield, R. C., Latushko, S. M. 2001, ApJ, 549, L261

Pevtsov, A. A., Canfield, R. C., Metcalf, T. R. 1994, ApJ, 425, L117

Pevtsov, A. A., Canfield, R. C., Metcalf, T. R. 1995, ApJ, 440, L109

Pevtsov, A. A., Canfield, R. C., Sakurai, T., Hagino, M. 2008, ApJ, 677, 719

Richardson, R. S. 1941, ApJ, 93, 24

Rust, D. M. 2003, Advances in Space Research, 32, 1895

Sankarasubramanian, K., Gullixson, C., Hegwer, S., et al. 2004, in Society of PhotoOptical Instrumentation Engineers (SPIE) Conference Series, eds. S. Fineschi \& M. A. Gummin, Society of Photo-Optical Instrumentation Engineers (SPIE) Conference Series, 5171, 207

Sankarasubramanian, K., Lites, B., Gullixson, C., et al. 2006, in Astronomical Society of the Pacific Conference Series, eds. R. Casini \& B. W. Lites, Astronomical Society of the Pacific Conference Series, 358, 201

Seehafer, N. 1990, Solar Phys., 125, 219

Sokoloff, D., Zhang, H., Kuzanyan, K. M., et al. 2008, Solar Phys., 248, 17

Tiwari, S. K., Joshi, J., Gosain, S., Venkatakrishnan, P. 2008, in Turbulence, Dynamos, Accretion Disks, Pulsars and Collective Plasma Processes, eds. S. S. Hasan, R. T. Gangadhara, \& V. Krishan, 329 